\author{
e-Journal of Educational \\ Research, Assessment and \\ Evaluation
}

Revista ELectrónica de Investigación y EValuación Educativa

\title{
EL PODER EDUCATIVO DE LAS ASOCIACIONES DE INMIGRANTES EN LAS ESCUELAS MULTICULTURALES
}

\section{[The education power of immigrant associations in multicultural schools]}

\author{
por/by
}

\author{
$\underline{\text { Article record }}$ \\ About authors \\ HTML format
}

\author{
Soriano-Ayala, Encarnación (esoriano@ual.es) \\ González-Jiménez, Antonio J. (ajgonzal@ual.es)
}

\author{
Ficha del artículo \\ $\underline{\text { Sobre los autores }}$ \\ Formato HTML
}

\begin{abstract}
With this qualitative research we are trying to know the collaborative capacity and contributions that Immigrant Associations could do to the schools that form part of a social context characterised by the recent and massive arrival of immigrants of Maghrebian, Sub-Sahara, South America, European Union, and East Europe origin. The sample, constituted by 55 immigrants that are members of Immigrant Associations, 16 teachers and 16 directors of schools, makes us to think about the role the Immigrant Associations could play in the education centres. The information, coming out from immigrants and teachers shows up that the participation of associations, besides to do a good intercultural work and favour the identity signs of new students, empower the school influence of immigrant children. In addition to, this participation would support the continuity between the school and the student family; it also constitutes a way of working with children and young people in communities of learning.
\end{abstract}

\section{Keywords}

Immigrant Associations, Intercultural Education, Intercultural Mediation, Empowerment, communities of learning.

\section{Resumen}

Con esta investigación cualitativa pretendemos dar a conocer la capacidad colaborativa y las aportaciones que pueden realizar las asociaciones de inmigrantes a las escuelas que forman parte de un contexto social caracterizado por la llegada reciente y masiva de inmigrantes de orígenes Magrebí, África Subsahariana, Sudamérica, Unión Europea y Europa del Este. La muestra constituida por 55 inmigrantes miembros de asociaciones de inmigrantes y 16 profesores y 16 directores de centros educativos, nos permite comprender el papel que pueden jugar las asociaciones de inmigrantes en los centros educativos. Los datos obtenidos a partir de los propios inmigrantes y profesores, ponen de manifiesto que la participación de las asociaciones, además de realizar una buena labor de mediación intercultural y favorecer las señas identitarias de los nuevos escolares, potencia el "poder" escolar y social de niños y jóvenes inmigrantes. Además, esta participación favorece la continuidad entre centro educativo y familias, y constituye una forma de poder trabajar con los niños y jóvenes en comunidades de aprendizaje.

\section{Descriptores}

Asociaciones de inmigrantes, educación intercultural, mediación intercultural, empoderamiento, comunidades de aprendizaje. 
En la sociedad postmoderna las oleadas rápidas de emigrantes/inmigrantes no constituyen un fenómeno nuevo, lo novedoso es su magnitud y las estrategias de adaptación. En la época actual aproximadamente 200 millones de personas viven fuera de sus países de origen. Es como si todo el mundo estuviera en todo el mundo, lo que supone que personas con diferentes referentes culturales y sociales están compartiendo espacio y tiempo (Soriano, 2004).

Con la llegada de personas de distintas procedencias culturales España, que ha sido históricamente un país de emigración, ha comenzado una transformación demográfica que tendrá unas profundas implicaciones en el futuro de este país (Soriano, 2006).

La década de los años setenta marcan un importante punto en la historia de la migración española: el número de españoles que emigran a trabajar a otros países decrece significativamente, mientras que el número de personas extranjeras que llegan a España buscando un trabajo y una forma de vida más digna se incrementa. En 1975 había en España 165.000 residentes extranjeros, en 1992 el número de adultos extranjeros alcanza la cifra de 415.000, a finales de diciembre de 2007 el número de extranjeros con certificado de registro o tarjeta de residencia en vigor ascendía a 3.979.014 (Ministerio de Trabajo y Asuntos Sociales, 2008) y, según la Secretaría de Estado de Inmigración y Emigración, a fecha de 31 de diciembre de 2008, el número de extranjeros ya asciende a 4.473 .499 (Ministerio de Trabajo e Inmigración, 2009).

La sociedad española cada vez es más plural, y coincidimos con Habermas (1999: 94,96) en destacar que en España hay "evidencias cotidianas que se alejan del caso modélico de un Estado nacional con una población culturalmente homogénea. Aumenta la multiplicidad de formas de vida, grupos étnicos, confesiones religiosas (...) Los ciudadanos deben poder experimentar el valor de uso de sus derechos también en la forma de segu- ridad social y de reconocimiento recíproco de las diferentes formas de vida culturales”. Esta circunstancia da lugar a que en España se ponga de relieve nuevas formas de autoorganización de los ciudadanos (Mezzana, 2003). Surgen nuevas entidades de ciudadanos y, entre ellas, las asociaciones de inmigrantes.

Las asociaciones de inmigrantes constituyen un espacio donde se fomenta la solidaridad y se apoya la construcción de la identidad cultural del inmigrante entre el país de origen y la tierra de adopción (Sipi, 2000). En las asociaciones los inmigrantes intercambian sus experiencias en el nuevo ciclo de vida que han iniciado, lo que supone un reencuentro con su país de origen. En el seno de las asociaciones se producen mediación y dialogo entre los inmigrantes.

Generalmente, las asociaciones de inmigrantes presentan una serie de características fundamentales:

- Constituyen un espacio de encuentro entre inmigrantes para expandir su cultura, costumbres y valores. Esta función favorece el conocimiento mutuo, necesario para superar los prejuicios sociales (Fernández y otros, 2001).

- Apoyan la afirmación de sus identidades y comunidades lo que favorece el desarrollo de identidades colectivas, la convivencia humana y satisface la necesidad de estar juntos en un lugar donde se sienten extraños (Pérez y López, 2003).

- Supone un ejercicio de la ciudadanía activa para mejorar la calidad de vida del inmigrante en el país anfitrión (González, 2005).

Las asociaciones de inmigrantes también tienen una función educadora y socializadora (González, 2005); transmiten valores de solidaridad, filantropía y tolerancia; constituyen un espacio para sensibilizar e impactar a la opinión pública y política sobre los problemas sociales de los inmigrantes y aumentan la sensibilidad de los ciudadanos ante los problemas de éstos. 
Desde el año 1994 nuestro grupo de investigación lleva a cabo investigaciones en las escuelas en las que hay escolarizados estudiantes autóctonos e inmigrantes. Como investigaciones más representativas llevadas a cabo en los primeros diez años señalaremos la investigación ${ }^{1}$ llevada a cabo en 1994 en la que se analiza la situación escolar de los niños y niñas magrebíes y subsaharianos escolarizados en la comarca del poniente almeriense. En 1998, el proyecto europeo ${ }^{2}$ realizado entre tres países en el que se trabaja con el profesorado de Educación Primaria diseñando estrategias y materiales de intervención en Educación Intercultural. Durante el curso académico 2000/01, dentro de un proyecto más amplio de investigación, se analiza la educación y la escolaridad de los niños/as y jóvenes inmigrantes en Andalucía ${ }^{3}$. Diez años más tarde, en 2004, a petición de Asociaciones de Inmigrantes senegalesas ubicadas en la provincia de Almería, comienza nuestra colaboración con estas Asociaciones. Al principio se mantienen reuniones con siete asociaciones de Senegal. De estos encuentros surge un proyecto de investigación en cooperación con las asociaciones, en el que se estudia la situación de este colectivo en Andalucía ${ }^{4}$. El acercamiento a las Asociaciones nos permite saber que en los cargos representativos tienen a personas con un buen nivel académico, muchas de ellas con estudios universitarios que han cursado en sus lugares de origen. Por otra parte, a las escuelas españolas están llegando un importante número de niños y jóvenes de diferentes procedencias (magrebíes, africanos subsaharianos, sudamericanos, europeos del este, asiáticos). En las investigaciones realizadas hasta ahora hemos observado que los profesores siempre se han quejado de desconocer la cultura de los nuevos alumnos, sus formas de vida, su lengua, su nivel curricular (Soriano, 1998, 2003, 2008a), a lo que se suma, además, el distanciamiento de la escuela que tienen las nuevas familias (Soriano, 2007, 2008b).

El conocimiento que tenemos de las Asociaciones de Inmigrantes y de la situación en la que hoy se encuentra la escuela en España, nos invita a estudiar un posible acercamiento entre las Asociaciones y la Institución escolar para que se establezca colaboración entre ambas.

La revisión de la literatura no nos muestra que se haya llevado a cabo un trabajo colaborativo de las Asociaciones de Inmigrantes con las escuelas, lo que nos hace reflexionar sobre cómo podría llevarse a cabo este acercamiento, de qué forma podrían colaborar y si tanto Asociaciones como profesores estarían dispuestos a ello. Por ello, el objetivo de esta investigación es conocer y comprender desde la perspectiva del profesorado y de las Asociaciones de inmigrantes cuales son las razones que justifican a participación de las Asociaciones de inmigrantes en los centros educativos multiculturales y cómo se puede establecer la colaboración entre ellas y el profesorado.

Entendemos que la colaboración de personas de las Asociaciones con la escuela, ayuda a entablar un diálogo intercultural con el entorno del joven inmigrante y también constituye una vía para el empowerment del alumnado inmigrante en la nueva sociedad.

\section{La perspectiva integradora y crítica de la educación}

La educación hemos de entenderla como un proyecto colectivo en un marco social, reflexivo e intencional que tiene puesta su mirada en un futuro mejor para el alumnado de todas las etapas educativas y de cualquier procedencia cultural. Por ello, es urgente ampliar el concepto de educación que se maneja actualmente y buscar soluciones que satisfagan las necesidades educativas del conjunto de la población ya sean autóctonos o procedentes de otras etnias y culturas (Soriano, 2005).

El capital social disponible de los estudiantes, su origen cultural y su historia y los contextos educativos que sirven a ellos, junto al estatus 
socioeconómico son factores cruciales para los resultados académicos de los diferentes estudiantes en la escuela (Portes, 1999). Además, sabemos que la relación entre familia y escuela es positiva, porque mejora el éxito escolar del alumnado, por tanto, es importante el encuentro entre los valores de la familia y las expectativas del profesorado (Henderson y Mapp, 2002). Pero, las percepciones de los padres y madres de cómo la escuela prepara a los niños y niñas varía según la raza, etnia y grupo social (Desimore, 1999). En la escuela del país de acogida, donde las familias no suelen visitarla porque ocupan mucho tiempo en el trabajo y desconocen la lengua para comunicarse con el profesorado, las Asociaciones de Inmigrantes pueden ejercer una labor de acercamiento entre escuela y familia.

Greene (1986) entiende la educación como comprensión orientada a dar poder a las personas para que se produzca un cambio en ellas, para que piensen critica y creativamente, para que tengan interés por conocer e incrementen el sentido que tienen sus vidas en el mundo. Piensa que el conocimiento es esencial para adquirir poder.

Cummins (1986) argumenta que los estudiantes pueden ser "empoderados" o "incapacitados” por el profesorado y las escuelas que sólo reflejan la cultura dominante de la sociedad. En un estudio que llevó a cabo con estudiantes negros, concluyó que la educación da poder si en los programas educativos la lengua y la cultura del otro queda incluida, si la comunidad participa de forma colaborativa y si la pedagogía se orienta hacia la interacción recíproca.

Dice Sleeter (1991) que una educación que potencie el empoderamiento (empowerment) del estudiante, tiene que trabajar con ellos y su comunidad para construir sobre lo que ellos ya aportan a la escuela. Nunca hemos de confundir educación de los miembros más desfavorecidos de la escuela con educación compensatoria. Los niños, las niñas y jóvenes inmigrantes que llegan a la escuela no necesitan compensar nada. Ellos aportan a la escuela una cultura que no es la escolar, ya que poseen una serie de conocimientos previos de fuera de la escuela, motivaciones, metas, estrategias para el aprendizaje, pertenencias e identidades personales que dirigen su crecimiento. Como dice Sleeter (1991) no necesitan una ayuda benevolente sino que la escuela tenga en cuenta estos conocimientos y construya a la persona desde lo que ya son. Como indican Shor y Freire (1987) el conocimiento que da poder está situado dentro e interactúa con la lengua y cultura de los estudiantes.

Creemos que la colaboración con la escuela de las Asociaciones de Inmigrantes que conocen y representan la cultura de los jóvenes inmigrantes hace que el profesorado vea al inmigrante como miembro de una cultura y con un bagaje cultural sobre el cual hay que empezar a construir significados y conocimientos. De esta forma se actúa con el alumnado en un doble sentido: el desarrollo de habilidades y capacidades para ayudarle a actuar con eficacia (Ashcroft, 1987) y, el fomento del aprendizaje crítico del conocimiento existente fuera de sus experiencias inmediatas.

Además de todo lo anterior, en el nuevo contexto actual de la sociedad de la información, marcada por la globalización y los movimientos migratorios, consideramos necesario que el aprendizaje sea un proceso intrínsecamente social, y coincidimos con Vila (2004) cuando afirma que para que tenga lugar el aprendizaje en la escuela, ésta debe realizar su trabajo en continuidad con los otros agentes educativos. Y no sólo eso: debe ser la escuela quien ha de ejercer un papel vertebrador y centrar con los agentes educativos tales como la familia, medios de comunicación, localidad, barrio, asociaciones, lugares de ocio, etc.

Es deseable, que en los contextos multiculturales, las escuelas se conviertan en auténticas comunidades de aprendizaje en donde se establezca una genuina correlación entre lo que ocurre en el aula, en casa, en la comunidad más cercana y en la información que llega a través de Internet y los medios de comunicación de masas. La escuela debe dejar de ser 
monolítica y anclada en el tiempo y ser coherente con la realidad social que le toca vivir.

\section{Metodología}

\section{El contexto de la investigación}

Esta investigación se lleva a cabo en Almería capital y provincia. Las escuelas almerienses escolarizan alumnado procedente de la inmigración desde 1990, siendo los primeros recién llegados de origen marroquí. Con la llegada de familias inmigrantes de diferentes procedencias, en las escuelas almerienses hallamos alumnado de origen magrebí, de África subsahariana, de Sudamérica, del este, del norte y del centro de Europa. En este contexto encontramos centros educativos de primaria y secundaria en los que el alumnado inmigrante supera el 60\%.

\section{Objetivos y participantes en el estudio}

Como hemos anotado en la introducción, refiriéndonos a investigaciones realizadas anteriormente, el profesorado se queja del desconocimiento que posee de la cultura del otro, del desconocimiento del idioma y de la falta de integración de muchos de los recién llegados. A ello, añadiremos los resultados hallados en el informe PISA sobre rendimiento académico del alumnado inmigrante y, en esta situación, entendemos que el trabajo colaborativo escuela- Asociaciones puede favorecer a mejorarla.

El objetivo principal de esta investigación es conocer y comprender desde la perspectiva de las Asociaciones de inmigrantes y del profesorado cuales son las razones que justifican la participación de las Asociaciones de inmigrantes en los centros educativos multiculturales y cómo se puede establecer la colaboración entre ellas y el profesorado.

Para alcanzar este gran objetivo, hemos entendido que la metodología más idónea es la cualitativa. Se trata de una investigación descriptiva de carácter comprensivo a partir de la visión de 62 personas inmigrantes miembros de 16 Asociaciones, 16 profesores y profesoras, y 16 directores y directoras de centros de infantil y primaria y secundaria; triangulando los resultados.

Los informantes de esta investigación, son miembros de las Asociaciones de Inmigrantes ubicadas en la ciudad de Almería y las comarcas del poniente y levante almeriense, y profesorado de centros educativos con alumnado inmigrante escolarizado y que en su entorno hubiera ubicada una asociación de inmigrantes.

Las Asociaciones de inmigrantes con las que entablamos relación son reconocidas por la Consejería de Justicia y gozan o han gozado de subvenciones públicas, lo que las hace estar activas.

La investigación se desarrolla en tres fases:

$1^{a}$ Fase: La perspectiva de las asociaciones de inmigrantes

\section{Objetivo especifico}

- Conocer, desde el punto de vista de las asociaciones de inmigrantes, las razones que justifican la participación de las asociaciones de inmigrantes en los centros educativos y cómo están dispuestas a colaborar.

\section{Participantes del estudio}

Los informantes de esta fase han sido 55 inmigrantes pertenecientes a 16 Asociaciones de Inmigrantes. Las Asociaciones están ubicadas en la ciudad de Almería y en las comarcas del poniente y del levante almeriense. Estas Asociaciones están inscritas en la Consejería de Justicia y gozan o han gozado de subvenciones públicas, lo que las hace estar activas. De los 55 participantes, 48 son entrevistados y 7 forman parte de un grupo de discusión.

- 48 inmigrantes han sido informantes a través de entrevistas. Por cada Asociación se han entrevistado a 3 personas. Las 16 Asociaciones de Inmigran- 
tes que han participado en las entrevistas son las siguientes:

3 Asociaciones de Inmigrantes procedentes de Marruecos.

1 Asociación de Mali

2 Asociaciones de Senegal.

1 Asociación de Guinea Bissau.

1 Asociación de Guinea Conakry.

1 Asociación de Ghana.

2 Asociaciones de Rusia.

1 Asociación de Mauritania.

1 Asociación de Pakistán.

1 Asociación compuesta por inmigrantes de diferentes nacionalidades.

Una federación de Asociaciones de Inmigrantes.

Una Asociación formada por inmigrantes procedentes de África.

- 7 inmigrantes participantes en el grupo de discusión. Un representante de cada una de las Asociaciones siguientes: Mujeres marroquíes, Senegal, Guinea Bissau, Ghana, Rusia, Asociación de trabajadores de inmigrantes marroquíes (ATIME) y Marruecos.

\section{$2^{a}$ Fase: La perspectiva del profesorado}

\section{Objetivos específicos}

- Conocer desde la perspectiva del profesorado las razones que justifican la participación de la Asociaciones de inmigrantes en las escuelas.

- Identificar en qué aspectos del currículum pueden participar las Asociaciones de inmigrantes para optimizar la integración y el éxito escolar del alumnado inmigrante.

- Valorar la colaboración con la escuela de las Asociaciones de Inmigrantes para atender educativamente al niño y joven inmigrante.

\section{Participantes del estudio}

Los informantes de esta fase los forman 16 directores y 16 profesores de escuelas de Edu- cación Infantil y Primaria, e Institutos de Educación Secundaría, ubicados en zona de máxima inmigración y que disponen en sus cercanías de al menos una Asociación de Inmigrantes.

$3^{a}$ Fase: La triangulación de ambas perspectivas

\section{Objetivo específico}

- Contrastar la información obtenida procedente de las Asociaciones de Inmigrantes, del profesorado y de los directores y directoras.

\section{Técnicas de recogida de datos y análisis de datos}

Los objetivos especificados en las fases del estudio se desarrollan realizando entrevistas en profundidad a representantes de las Asociaciones de Inmigrantes, y a directores/as y profesorado. Completando las entrevistas se lleva a cabo un grupo de discusión con inmigrantes pertenecientes a Asociaciones.

Antes de realizar entrevistas, hemos visitado y tenido contacto con cada una de las asociaciones que han aceptado participar en esta investigación, y con los participantes de los centros educativos

Se elaboran dos guiones orientativos de preguntas abiertas para las Asociaciones y el profesorado con ejes similares de contenidos. La aplicación de las entrevistas se han llevado a cabo en tres sesiones:

- En la primera sesión, pretendíamos recabar toda la información relevante e importante sobre el guión orientativo de preguntas.

- En la segunda sesión, formulábamos dudas o lagunas informativas que nos habían surgido durante las transcripciones y lectura de las entrevistas realizadas.

- En la tercera sesión y una de la más importante, desde nuestro punto de 
vista, consistió en leerles a los participantes entrevistados los diversos informes procedentes de las entrevistas para que subsanaran los errores de los mismos. De esta manera, validábamos la información recogida.

Una vez analizada la información derivada de las entrevistas en profundidad, se organizó un grupo de discusión. La utilización de esta técnica como recogida de datos, se fundamenta en validar las diferentes opiniones y conclusiones extraídas en las entrevistas. En el grupo de discusión participan 7 representantes de las Asociaciones de Inmigrantes.

Las entrevistas y el grupo de discusión se graban, se trascriben, se buscan categorías y se codifican. El análisis de datos se lleva a cabo con el programa de análisis de datos cualitativos AQUAD 6.0

Finalmente se contrastan las conclusiones derivadas de las estrategias de recogida de datos a las Asociaciones de Inmigrantes, y del profesorado y directores/as de los centros educativos.

La investigación cumple los criterios de rigor científico de la investigación cualitativa. La credibilidad queda probada por la estrategia que se lleva a cabo de entrevistar y devolver la entrevista transcrita al entrevistado para que la complete o subsane los errores y, además, por la estrategia de triangulación. La transferibilidad se ha conseguido a través del muestreo teórico y por la recogida abundante de información. Las estrategias de triangulación y los descriptores de baja inferencia como es el caso de las transcripciones textuales nos permite alcanzar la confirmabilidad.

En los dos cuadros siguientes aparecen los sistemas de categorías utilizados en las entrevistas y grupo de discusión llevado a cabo con las Asociaciones de Inmigrantes, y con el profesorado y directores/as.

Cuadro 1.Sistema de categorías de las razones que justifican la participación de las asociaciones de inmigrantes en los centros educativos.

\begin{tabular}{|c|l|}
\hline $\begin{array}{c}\text { Agentes de la } \\
\text { comunidad educativa }\end{array}$ & Profesorado \\
& Familias inmigrantes \\
& Familias autóctonas \\
& Alumnado inmigrante \\
& Alumnado autóctono \\
\hline
\end{tabular}


Cuadro 2. Sistema de categorías de las propuestas de participación de las Asociaciones de Inmigrantes en el centro educativo.

\begin{tabular}{|c|c|c|}
\hline Currículum & \multicolumn{2}{|c|}{$\begin{array}{l}\text {-Actividades interculturales con padres autóctonos } \\
\text {-Actividades interculturales con padres inmigrantes } \\
\text { - Asociación de padres inmigrantes } \\
\text { - Actividades de tutoría } \\
\text { - Departamento de orientación } \\
\text { - Ejes transversales } \\
\text {-Documentos curriculares }\end{array}$} \\
\hline \multirow{5}{*}{ Agentes educativos } & Alumnado inmigrante & $\begin{array}{l}\text {-Actividades de acogida } \\
\text {-Mediación alumno inmigrante-profesorado } \\
\text {-Integración inmigrante }\end{array}$ \\
\hline & Alumnado autóctono & $\begin{array}{l}\text {-Aceptación del inmigrante } \\
\text {-Conocimiento de la cultura del otro } \\
\text {-Convivencia entre culturas } \\
\text {-Propuestas de actuación }\end{array}$ \\
\hline & Familias inmigrantes & $\begin{array}{l}\text {-Cambio de actitudes hacia el sistema educativo } \\
\text {-Asesoramiento } \\
\text {-Mediación entre centro y familias }\end{array}$ \\
\hline & Familias autóctonas & $\begin{array}{l}\text {-Cambio de actitudes } \\
\text {-Fomentar la convivencia } \\
\text {-Acercamiento a las familias inmigrantes }\end{array}$ \\
\hline & Profesorado & $\begin{array}{l}\text {-Facilitar recursos culturales } \\
\text {-Enseñanza de otra cultura } \\
\text {-Mediación con padres inmigrantes } \\
\text {-Características del inmigrante }\end{array}$ \\
\hline
\end{tabular}

\section{Resultados}

Los resultados que exponemos proceden de la triangulación metodológica de la información de las entrevistas en profundidad y el grupo de discusión llevado a cabo con las asociaciones de inmigrantes, y por otro lado, las entrevistas en profundidad realizadas al profesorado.

Para su comprensión, organizamos los resultados en los aspectos que hemos considerado fundamentales para responder a nuestros objetivos de investigación.

¿Cuáles son las razones que justifican la participación de las Asociaciones de Inmigrantes en los centros educativos? El punto de vista de las propias asociaciones

Las principales razones que exponen nuestros informantes y representantes de las Asociaciones de Inmigrantes para justificar su presencia en los centros educativos se centran en cuatro amplios agentes de actuación: con el profesorado, con las familias inmigrantes y autóctonas y con el alumnado inmigrante y autóctono.

\section{Profesorado}

En el análisis del discurso de los inmigrantes entrevistados, aparece de forma repetida la creencia de que muchos profesores y profesoras presentan resistencia a favorecer la interculturalidad en sus aulas y una escasa motivación para integrar al alumnado inmigrante. Se quejan de la ausencia en las aulas de actividades que tengan como objetivo fomentar la igualdad, la valoración del "otro" (al que consideran diferente) y de respetar la diferencia cultural. También se refieren a la falta de actividades de apoyo organizadas por la comunidad educativa fuera del horario escolar. Uno de los entrevistados perteneciente a una aso- 
ciación de inmigrantes de Senegal se expresa así: "... fundamentalmente son los padres y después los profesores los que no se esfuerzan por poner a trabajar de forma pedagógica a los niños autóctonos e inmigrantes en un mismo plano, es decir, en actividades que fomenten la igualdad, no sé cómo explicarme pero creo que se me entiende..." (AIS2) ${ }^{5}$.

Por estas razones proponen colaborar con el profesorado apoyándolos en el conocimiento de la cultura que configura parte de la identidad del alumnado inmigrante. Proporcionar al profesorado materiales y recursos para favorecer la interculturalidad en la escuela y ejercer labores de mediación con familias y alumnado.

\section{Familias de alumnado inmigrante}

A lo largo de la investigación apreciamos que los entrevistados reconocen explícitamente que el reto de integración del alumnado inmigrante no recae solamente en el profesorado o en la Institución Educativa, sino que también son responsables las familias inmigrantes debido a su baja participación en los centros educativos. Uno de los representantes de una asociación de inmigrantes de Pakistán lo expresa de esta forma "No se integran educativamente. También es culpa de los padres porque no se interesan por la educación de los hijos. Yo creo que desde las asociaciones debemos concienciar a los padres de la importancia de que colaboren en la educación de sus hijos"(AIP1) ${ }^{6}$. Entienden los inmigrantes que su actuación con el profesorado ayuda a involucrar a las familias inmigrantes en el proceso educativo de sus hijos e hijas. Además, una labor importante que pueden llevar a cabo es establecer lazos de unión entre familias y centro educativo.

\footnotetext{
${ }^{5}$ AIS2, segundo inmigrante entrevistado de la Asociación de Senegal.

${ }^{6}$ AIP1, primer inmigrante entrevistado de la Asociación de Pakistán.
}

\section{Familias de alumnado autóctono}

Las asociaciones de inmigrantes también subrayan el papel crucial que juegan las familias del alumnado autóctono en la transmisión de conductas discriminatorias y estereotipos hacia los inmigrantes. Por lo que es necesario diseñar acciones que permitan el acercamiento de los niños y jóvenes autóctonos al inmigrante. Nos dice uno de los miembros de una asociación rusa "Yo pienso que no es solamente racismo porque son muy chicos para comprender y llevar a cabo esa cosa, yo creo que el problema está en las familias, pues inculcan conductas negativas hacia los niños"(AIR3) ${ }^{7}$. Las Asociaciones proponen intervenir con familias autóctonas a través de la mediación para provocar cambios en las actitudes que tienen hacia el "otro". De esta forma pueden proporcionar a sus hijos e hijas una educación sana y desprovista de prejuicios.

\section{Alumnado inmigrante y autóctono}

Finalmente, los representantes de las Asociaciones de Inmigrantes entrevistados hablan de la existencia de problemas entre los inmigrantes y los autóctonos. Estos problemas son sobre todo de convivencia debido a los choques culturales que se dan entre inmigrantes y autóctonos, y entre los propios inmigrantes, puesto que proceden de países y culturas diferentes. Una mujer inmigrante marroquí habla de la siguiente forma "Es muy importante el acercamiento entre nosotros y los autóctonos. Es necesario hacer lo imposible para que se produzca ese acercamiento. Es la mejor forma de conocerse y quitar estereotipos; por ejemplo, el otro día llegó un chico de aquí y me dijo que no se esperaba ver a una mujer marroquí manejando el ordenador..." (AIM2) ${ }^{8}$. Las Asociaciones proponen intervenir con el alumnado autóctono a través de

\footnotetext{
${ }^{7}$ AIR3, tercer entrevistado perteneciente a una asociación rusa.

${ }^{8}$ AIM2, segunda entrevistada de una asociación de inmigrantes marroquíes
} 
campañas de sensibilización, y apoyando al profesorado en el diseño de actividades interculturales. Ven importante su presencia en la escuela porque conocen la cultura del joven inmigrante y pueden intervenir para solucionar los conflictos culturales. Por otra parte, entienden que su presencia en el centro educativo favorece el reconocimiento de su grupo cultural ante los ojos del alumnado inmigrante.

\section{¿Cuáles son las razones que justifican la participación de las asociaciones de inmi- grantes en los centros educativos? La per- cepción del profesorado}

El profesorado entrevistado coincide bastante con las opiniones de los representantes de las asociaciones de inmigrantes. Las razones que justifican la participación de las asociaciones de inmigrantes en los centros educativos, han sido clasificadas en función de los problemas que encuentran los docentes con los siguientes agentes de la comunidad educativa: alumnado inmigrante, alumnado autóctono, familias de alumnado inmigrante, familias de alumnado autóctono y el propio colectivo docente. Este apartado se complementa con el siguiente donde el profesorado expone cómo se debe llevar a cabo la participación en la escuela de las Asociaciones de Inmigrantes.

\section{Alumnado inmigrante}

El profesorado señala que la principal dificultad que tiene es el desconocimiento de la lengua materna de la mayoría del alumnado inmigrante que llega a sus clases, acentuado por la ausencia de mediadores interculturales que faciliten la comunicación entre ellos y el alumnado inmigrante. Hacen referencia, además, a la falta de integración de los alumnos inmigrantes con los autóctonos, al bajo dominio del español y al desfase escolar con el que llegan estos niños al centro educativo, así nos dice un profesor "Los niños tienen muchos prejuicios con los niños marroquíes, son más racistas y convivir en estos niveles cuesta, veríamos que no somos tan diferentes. Los niños verían que cada cultura e inmigrante tienen sus cosas, su música, sus danzas, sus bailes...Hay que tener más contacto con los inmigrantes y no exclusivamente en las jornadas interculturales... Necesitamos personas especializadas que nos ayuden como los mediadores interculturales..." (P5)

\section{Alumnado autóctono}

Para el profesorado el obstáculo fundamental presenciado en el alumnado autóctono es la falta de una verdadera convivencia, debido muchas veces al desconocimiento de las culturas y a la existencia de actitudes y estereotipos hacia los alumnos inmigrantes. En palabras de uno de los profesores refiriéndose a las Asociaciones de Inmigrantes "Claro, tienen que participar en los centros educativos para que los alumnos autóctonos vean al inmigrante como una persona más y no como un inmigrante. Me explico, un inmigrante puede llevar 15 años en España y siempre será un inmigrante, creo que eso es un problema que tiene la sociedad española"(P8).

\section{Familias de alumnado inmigrante}

El bajo interés que muestran las familias inmigrantes por la educación que están recibiendo sus hijos y la inexistente participación de éstas, es el principal problema que encuentra el profesorado en relación con las familias inmigrantes. Nos dice el director de uno de los centros "Les da igual, lo único que les interesa es que sus hijos estén escolarizados, no sé si por el asunto de regularización, pero cuando vienen citados porque hay algún problema con el alumno acuden con bastante dificultad, nos cuesta incluso localizarlos y parece que sí muestran interés pero la situación no mejora" (D1) ${ }^{10}$.

Otro problema vinculado con el anterior, es la falta de motivación por parte de los padres y madres para que sus hijos sigan los estudios 
no obligatorios y este problema se acentúa, cuando los padres de origen marroquí se niegan a que las niñas inmigrantes marroquíes prosigan estudios no obligatorios.

\section{Familias de alumnado autóctono}

Los docentes nos transmiten que las barreras más latentes con las familias del alumnado autóctono son las actitudes negativas que tienen a que sus hijos compartan centro educativo con alumnado inmigrante y la nula convivencia fuera del centro escolar entre autóctonos e inmigrantes. Además, se aprecia una gran desconexión con la cultura del joven inmigrante. Así habla un director:

“...que los padres autóctonos vean que por estar sus hijos con inmigrantes, no les va repercutir en el desarrollo académico. Los padres deberían participar más, participar para que la población autóctona viera que existen otros mundos y un mundo de convivencia es posible, se deben de romper fronteras y llegar a relaciones mundiales. También favorecería la integración de los colectivos de inmigrantes" (D7).

\section{Con el profesorado}

Los impedimentos más frecuentes que presenta el colectivo docente son la falta de tiempo para buscar materiales y recursos originarios de la cultura del alumnado inmigrante, el desconocimiento de las culturas existentes en el aula, la falta de participación y conexión de los padres y madres inmigrantes con ellos y la ausencia de información sobre las circunstancias personales (económicas, sociales, familiares...) del alumno inmigrante. En palabras de un profesor "En muchas ocasiones el profesorado desconoce las otras culturas y no dispone de suficiente tiempo para buscar materiales. También es difícil porque el profesorado desconoce las costumbres y la cultura de ese alumnado, con lo cual nos dificulta el saber. Por ejemplo, los primeros años yo recuerdo que para nosotros era una sorpresa lo que era la fiesta del cordero para los musulmanes y había niños que no venían a clase, nos parecían extrañas ciertas conductas. Hoy en día nos parece lo más normal” (P2).

\section{¿Cómo pueden participar las Asociaciones de Inmigrantes en las escuelas? Propuestas de participación según los profesores}

Las siguientes propuestas o vías de participación de las Asociaciones de Inmigrantes en las escuelas, son las respuestas a las justificaciones que en el apartado anterior hace el profesorado. Las propuestas han sido organizadas en dos ámbitos: en el currículum y con los agentes educativos.

\section{Ámbitos de participación en el currículum}

El profesorado entrevistado propone la participación de las asociaciones de inmigrantes en diferentes ámbitos del currículum. Las propuestas son las siguientes:

- En las Asociaciones de Madres y Padres. Señalan que las Asociaciones de Inmigrantes harían una buena labor participando mediante la proposición de diversas actividades interculturales o bien formar sus propias asociaciones de padres y madres inmigrantes, lo que desde nuestro punto de vista, si se realiza esto último, favorecerían el segregacionismo de los padres y madres.

- En las Actividades de Tutoría y Departamento de Orientación. Los docentes entrevistados nos indican que la participación de las asociaciones de inmigrantes sería un instrumento de gran valor a la hora de proponer actividades interculturales a los tutores y al Departamento de Orientación.

- En los Ejes Transversales. El profesorado nos sugiere que las asociaciones de inmigrantes pueden colaborar en los ejes transversales, concretamente 
en el eje transversal de Educación para la Paz y la Convivencia mediante el diseño y desarrollo de actividades.

- En la elaboración de los Documentos Curriculares. Otra posible participación de las Asociaciones, según el profesorado, es en el diseño de las comisiones de convivencia, en los Proyectos Educativos de Centro y en los Proyectos de Orientación y Acción Tutorial.

Participación con los agentes de la comunidad educativa

\section{Con el alumnado inmigrante}

El profesorado coincide en una serie de propuestas de participación de las Asociaciones de Inmigrantes con el alumnado inmigrante. Dichas propuestas de intervención han sido agrupadas en tres ámbitos:

- Acogida al alumnado inmigrante. Sugieren que las asociaciones colaboren en las actividades de acogida al alumno inmigrante al principio de curso con la finalidad de explicarles a los estudiantes el funcionamiento del centro.

- Mediación entre alumnado inmigrante y profesorado. El profesorado propone que sean las Asociaciones de Inmigrantes las que actúen como mediadoras con los diversos obstáculos que se encuentran los docentes al desarrollar su labor educativa con el alumnado inmigrante.

- Integración escolar. Plantea el profesorado que las Asociaciones de Inmigrantes colaboren en el diseño de actividades que fomenten la integración del alumnado inmigrante en el ámbito escolar, tales como:

1) Cursos de aprendizaje de español llevadas a cabo por las propias Asociaciones en el centro educativo, pero en hora extraescolar. Un profesor explica en las siguientes líneas la impor- tancia del aprendizaje de español: “... muchos de los niños vienen sin saber español y las Asociaciones deberían tratar de ayudarnos a que aprendan español. Cuanto antes el niño aprenda, antes podrá integrarse en la sociedad y en el colegio, por ejemplo, clases de repaso, de español... Aquí podría venir gente inmigrante que no fueran alumnos matriculados del centro para dar clases de español”(P11).

2) Actividades que fomenten la autoestima y el cambio de actitudes del alumnado inmigrante. Este tipo de actividades nos llamó bastante la atención debido a su finalidad y porque coincide con las aportaciones de Cummins (1986) y que hemos señalado en la parte teórica. El profesorado defiende la idea de que las actividades organizadas e impartidas por las propias Asociaciones de Inmigrantes fomentarán la autoestima y el cambio de actitudes del alumnado debido a que percibirá que su colectivo es valorado y que puede realizar importantes aportaciones al funcionamiento del centro educativo, es decir, no se verán como una minoría étnica sometida a los dictámenes del grupo autóctono. La importancia de la autoestima y del cambio de actitudes en el alumnado inmigrante, la explica uno de los profesores de la siguiente forma:

"Es importante que el alumno vea que su cultura es valorada, por ejemplo, la Asociación puede venir y realizar algún tipo de actividad sobre su cultura, entonces el alumno inmigrante ve que su cultura se valora porque los alumnos autóctonos participan, preguntan y se interesan. En definitiva, le damos protagonismo a este sector, se rompe el predominio de la cultura española. Es un referente porque los alumnos inmigrantes verán que 
son personas que no solamente vienen al colegio para recibir, sino también para dar. De esta forma, no se verán como intrusos que vienen aquí para que se lo den todo, que no saben nada y simplemente son ejecutores de directrices, se verán como personas que pueden aportar cosas” (P15)

3) Actividades de convivencia entre los diversos colectivos de inmigrantes y el alumnado autóctono. Esta forma de participación basada en la convivencia es narrada por dos entrevistados: "Cuanto mayor sea la participación de las asociaciones en los centros educativos, mayor será la integración de los alumnos inmigrantes.jMira!, el contacto entre personas "diferentes" hace mucho, hace que cambien nuestros estereotipos y conductas, también hacen que hablemos bien a otras personas de nuestra propia cultura sobre los inmigrantes” (P16); “...la participación de las asociaciones favorecerá el cambio de actitudes de los niños autóctonos hacia los inmigrantes. Pienso que el conocimiento y acercamiento a la cultura ajena, es una herramienta poderosa de cambio de actitudes. Además, los alumnos inmigrantes también cambiarían sus actitudes hacia los españoles porque apreciarían que su cultura es valorada por el profesorado $y$ por sus compañeros"(D2)

4) Actividades de refuerzo educativo. Las propias Asociaciones deberán organizar actividades de refuerzo escolar para el alumnado inmigrante que presenta un marcado desfase escolar

\section{Con el alumnado autóctono}

El profesorado coincide en la idea de que las asociaciones de inmigrantes tienen que organizar actividades destinadas al alumnado autóctono para que acepten al inmigrante como un ciudadano más, conozcan su cultura y cos- tumbres, se fomente la convivencia y conocimiento entre culturas y el cambio de actitudes y prejuicios por parte del autóctono hacia el inmigrante, se produzca acercamiento entre los diferentes grupos culturales... Uno de los entrevistados nos dice: “...un inmigrante puede llevar 15 años en España y siempre ser un inmigrante, creo que eso es un problema que tiene la sociedad española. Las Asociaciones tienen que colaborar para que los alumnos autóctonos vean al inmigrante como una persona con sentimientos igual que ellos, y no únicamente como inmigrantes, es decir, que los vean como sujetos y no como inmigrantes, extraños...” (P8).

Algunas de las propuestas que nos sugiere el profesorado para que sean realizadas por las Asociaciones, relacionadas con el estudiante autóctono, son las siguientes: 1) Exposiciones orales por parte de algunos dirigentes de las Asociaciones para que expliquen en las escuelas su historia de vida, es decir, por qué emigraron, características sociolaborales y económicas de sus países, primeros años en España... El profesorado defiende la idea de que este tipo de actividades en las que un inmigrante narra las circunstancias de su proceso migratorio favorece el cambio de actitudes y prejuicios que los autóctonos tienen hacia el inmigrante, 2) Actividades donde se fomenten la integración y la convivencia entre el alumnado de diversos grupos culturales, incluido el "autóctono", y 3) Actividades en las que se expliquen y se difundan los rasgos principales de su cultura.

\section{Con las familias inmigrantes}

Según el profesorado la participación de las Asociaciones con las familias inmigrantes se centran en dos aspectos fundamentales:

1. Tratar de cambiar las actitudes de estas familias hacia el Sistema Educativo. Algunas de las labores que establece el colectivo docente para conseguir este objetivo son: 
- Explicar a las familias la importancia que tiene para sus hijos e hijas terminar los estudios obligatorios, y en su caso, seguir estudiando.

- Informar a los padres y a las madres de las características de la educación que están recibiendo sus hijos e hijas en el nuevo país.

- Motivar a los padres y a las madres para que inculquen a sus hijos e hijas hábitos de estudio y de responsabilidad hacia su aprendizaje.

- Dar a conocer a las familias aspectos relacionados con la cultura del país anfitrión.

- Asesorar a las familias acerca de lo importante que es su relación con la escuela, asistiendo a las tutorías para informar al profesorado sobre los problemas que tienen sus hijos e hijas, para recabar información sobre el progreso educativo de sus hijos e hijas, y para colaborar con el profesorado.

2. Puente de unión entre los centros educativos y las familias de alumnado inmigrante. Los docentes recalcan que un cambio de actitudes en las familias inmigrantes para que se interesen por la educación que están recibiendo sus hijos e hijas, tiene que ir acompañado por un aumento de la participación de los padres y de las madres en los centros educativos. Algunas de las propuestas para esta finalidad, son las que a continuación detallamos:

- Los padres y las madres pueden participar en los centros educativos dando charlas al alumnado autóctono sobre aspectos relacionados con sus países de origen.

- Las familias pueden dar a conocer a las niñas y niños autóctonos su lengua materna.

\section{Con las familias autóctonas}

Los profesores proponen que las Asociaciones de Inmigrantes intervengan con las familias autóctonas en dos aspectos:
- Cambio de actitudes. Los docentes señalan que las Asociaciones de Inmigrantes pueden realizar actividades de sensibilización que eliminaran las actitudes y prejuicios que tienen las familias autóctonas hacia la presencia de alumnado inmigrante en los centros educativos; por ejemplo, la creencia que se tiene que el alumnado inmigrante baja el nivel académico del centro.

- Fomentar la convivencia y acercamiento entre las familias autóctonas $e$ inmigrantes. Algunas de las actividades que proponen los docentes se basan en la realización por parte de las Asociaciones de Inmigrantes de actividades que fomenten la convivencia y el acercamiento de las familias inmigrantes a los autóctonos. Nos dice uno de los directores "No sería malo que hubiera una conexión entre padres. Es importante que ellos conozcan las diferentes culturas y se respeten. Yo creo que es ese el problema más importante. Que se conozcan las costumbres, las distintas religiones, las distintas maneras de ver la vida y que sean capaces de aportar lo bueno que tiene cada una de ellas y rechazar lo malo”(D3).

\section{Con los propios profesores}

El profesorado entiende que las Asociaciones de Inmigrantes pueden llevar a cabo una gran labor de apoyo a su docencia. Las propuestas se exponen en los siguientes ámbitos:

- Facilitar recursos de otras culturas. El profesorado señala que sean las propias Asociaciones las que se encarguen de concederles materiales propios de la cultura de los países del alumnado. En palabras de un profesor "Las asociaciones podrían traer literaturas de sus países para que los profesores las utilicemos, también es- 
critos y cosas que hacen. Si tenemos niños marroquíes, podemos trabajar con asociaciones de marroquíes para que nos traigan cosas. Rumania tiene ese periódico que te he dicho $y$, en el último, venía un libro que han hecho, está escrito por poetas rumanos en España y lo podemos utilizar” (P6).

- Enseñanza de las “otras” culturas. Este colectivo también sustenta la idea de que sean las propias Asociaciones las que les faciliten (a ellos mismos) el conocimiento de las connotaciones principales de la cultura de los alumnos inmigrantes. Un docente explica el beneficio cultural de la participación de las Asociaciones "Nos interesaría que las asociaciones participen para conocer la cultura de los alumnos inmigrantes. Porque en la enseñanza hay que partir de los conocimientos y experiencias de los niños para que avance la educación de forma positiva en los alumnos, por lo tanto, nos interesa saber cuál es su cultura..." (P4)

- Mediadores entre el profesorado y las familias inmigrantes. Un aspecto fundamental que recalcan todos los profesores a lo largo de las entrevistas, es la necesidad urgente de que sean las Asociaciones de Inmigrantes las que desarrollen funciones que propicien el acercamiento de los padres y madres inmigrantes a la vida escolar.

- Asesoramiento acerca de las características del alumnado inmigrante. En este aspecto, señalan que una vez que las Asociaciones se han aproximado a las familias de los alumnos inmigrantes, sean ellas las que informen al profesorado sobre las circunstancias que rodean a este alumno.

\section{Conclusiones}

Es muy valioso que tanto el profesorado como las Asociaciones de Inmigrantes, separadamente, justifiquen y argumenten razones muy similares para que las Asociaciones hagan aportaciones y participen activamente en el ámbito escolar.

Las conclusiones de este estudio son :

1. Existe disposición e interés por parte del profesorado y de las Asociaciones de Inmigrantes en colaborar y complementarse en acciones y actividades que mejoren la integración de los niños, las niñas y jóvenes inmigrantes.

2. Los docentes y las Asociaciones de Inmigrantes coinciden en los problemas que presentan el alumnado inmigrante y autóctono, el colectivo docente y las familias inmigrantes. También coinciden en la necesidad de que las Asociaciones de Inmigrantes participen en los centros educativos para la superación de estos obstáculos, mediante el desarrollo de una mediación intercultural, el acercamiento de las familias inmigrantes a los centros educativos y con actividades que fomenten la convivencia cultural.

3. La colaboración entre Asociaciones de Inmigrantes y profesores logrará que la escuela y la sociedad vean al alumnado inmigrante como portador de un capital social y cultural a partir del cual hay que comenzar la construcción de los conocimientos en el nuevo sistema educativo.

4. La colaboración en la escuela de las Asociaciones de Inmigrantes del entorno ayuda al empoderamiento (empowerment) de los jóvenes inmigrantes y autóctonos.

5. La educación y el aprendizaje son actos sociales que requieren procesos en los que haya continuidad entre los agentes educativos, uno de estos agentes es la Asociación de Inmigrantes. 
6. La participación de las asociaciones de inmigrantes en el aula y con los otros agentes de la comunidad educativa, favorecerá la creación de comunidades de aprendizaje.

La primera razón que argumenta las Asociaciones de Inmigrantes para ser agentes educativos es que perciben desmotivación en el profesorado para acoger en el aula a alumnado inmigrado, y carencia de actividades que potencien la interculturalidad. El profesorado, en cambio, se ve a sí mismo con falta de tiempo para atender a todas las necesidades que aparecen en un aula multicultural. Comenta el gran desconocimiento que posee de las culturas que el nuevo alumnado aporta al aula y observa que las familias inmigrantes están muy distanciadas del centro educativo. Estas razones invitan a intervenir en la escuela a las Asociaciones de Inmigrantes.

Las Asociaciones de Inmigrantes declaran razonadamente que ellas pueden actuar con las familias inmigrantes y autóctonas, consideración que también propone el profesorado. Las Asociaciones proponen sensibilizar a las familias inmigrantes para acercarlas al centro educativo y concienciar a los padres y madres para que asuman la responsabilidad que tienen en la integración de sus hijos e hijas en la nueva sociedad. Con las familias autóctonas, entienden que han de actuar para cambiar actitudes, puesto que transmiten a sus hijos e hijas estereotipos y conductas discriminatorias que les dificultan la convivencia con otras culturas. El profesorado coincide con las Asociaciones de Inmigrantes en señalar que éstas han de atender a familias autóctonas e inmigrantes. Con las autóctonas porque aprecian en ellas actitudes negativas a que sus hijos e hijas compartan Centro con los inmigrantes, y con las familias inmigrantes porque les falta motivación e interés por la educación de sus hijos. Coincidimos con Henderson y Mapp (2002) en la importancia que tiene el trabajo entre escuela y familia para la formación integral del alumnado y para que en el ámbito socioeducativo la convivencia entre grupos culturalmente diversos sea armónica.

La última justificación que da el profesorado y las Asociaciones para facilitar la entrada de éstas a las escuelas, es la labor que pueden llevar a cabo con el alumnado autóctono e inmigrante. Ambas entienden que los problemas vienen determinados por los choques culturales. Por lo que proponen, intervenir con el alumnado autóctono para que mejore la percepción que tiene del inmigrante, y con el alumnado inmigrante para facilitar su integración, subsanar su barrera idiomática y reforzar los conocimientos para paliar el fracaso escolar.

Hemos observado que las razones que el profesorado y las Asociaciones de Inmigrantes dan para facilitar el acceso de éstas a las escuelas son convincentes. También apreciamos la predisposición y la coincidencia de ambas partes en valorar positivamente esta participación.

El profesorado, que hasta ahora no ha trabajado en la escuela conjuntamente con las Asociaciones de Inmigrantes, percibe cómo debe plantearse esta colaboración. Para ellos una aportación importante de las Asociaciones de Inmigrantes es en las actividades de tutoría y en el currículum, diseñando conjuntamente documentos y materiales. Valoran positivamente la intervención de las Asociaciones en las actividades de acogida al alumnado inmigrante, su labor de mediación para favorecer la integración, la convivencia, y la mejora de la autoestima y el autoconcepto de los jóvenes inmigrantes. Piensan que, además, pueden actuar con el alumnado autóctono cambiando actitudes. Cosa que también deben hacer con las familias autóctonas. Mientras que con las familias inmigrantes pueden jugar un papel significativo mediando para que vean lo importante que es la educación para sus hijos e hijas.

Apreciamos que tanto el profesorado como las Asociaciones están dispuestas a colaborar entre ellos para que el alumnado inmigrante construya nuevos conocimientos y significa- 
dos teniendo en cuenta sus conocimientos previos, sus estrategias de aprendizaje y la identidad cultural que este alumnado ya traen cuando llegan al nuevo Sistema Educativo (Sleeter, 1991), y que son diferentes al del autóctono. Por otra parte, también percibimos el beneficio de la participación de las Asociaciones porque como concluyó Cummins (1986) en sus estudios, la educación da poder (empowerment) si considera y valora la lengua y la cultura del estudiante. Además, siguiendo a McLaren(1989) que define el propósito social del empowerment, la colaboración en la escuela de las Asociaciones de Inmigrantes logrará dar poder no sólo a los estudiantes inmigrantes, sino también a los autóctonos porque aprenderán críticamente de las experiencias no próximas, se ampliará la comprensión de los estudiantes autóctonos e inmigrantes y se transformarán las ideas, los prejuicios y el racismo que se percibe en muchos niños/as y jóvenes autóctonos hacia los inmigrantes, lo que favorecerá el diálogo intercultural.

Las Asociaciones de Inmigrantes, en su papel de mediadoras, lograrán un acercamiento entre la escuela y las familias, que como ha señalado Henderson y Mapp (2002) mejorará el valor que las familias otorgan a la escuela y el éxito escolar de los/as niños/as y jóvenes.

Entendemos, además, que la educación es sobre todo un proyecto colectivo, de colaboración, entre todos los implicados en la vida de un/una niño/a o joven: profesorado, familias, centros culturales, asociaciones culturales y, según nuestra investigación, las Asociaciones de Inmigrantes del entorno local. Como indica Vila (2004) la educación es un proceso eminentemente social y para que este llegue a realizarse debe haber una continuidad entre los agentes educativos. No debe recaer toda la responsabilidad sobre la escuela, hay que estar en comunicación y actuación con otros agentes educativos, en contextos multiculturales, uno de los agentes es las Asociaciones de Inmigrantes. Por otra parte, coincidiendo con Flecha y Puigvert (2008), entendemos que hay que movilizar los recursos existentes y mantener una postura abierta a la negociación y colaboración con otras personas dentro del aula. La colaboración de las Asociaciones de Inmigrantes dentro del aula y en el centro educativo favorecerá el aprendizaje dialógico transformador de la escuela y del entorno. Es ideal establecer comunidades de aprendizaje, haciendo colaborar a las Asociaciones de Inmigrantes en estos ambientes educativos multiculturales, para combatir situaciones de desigualdad a los que pueden llegar alumnado, autóctono e inmigrante, con riesgo de exclusión.

\section{Referencias}

Ashcroft, L. (1987). Defusing "empowering”: The what and the why. Language Arts, 64, 142-156.

Brunet Icart, I; Belzunegui Eraso, A y Pastor González, I. (2005). Problemática en torno a la integración de los inmigrantes en Educación Primaria: ¿desigualdades sociales o culturales?. Revista de Educación, 336, 293311.

Calvo, T. (2000). Inmigración y racismo. Así sienten los jóvenes del siglo XXI. Madrid: Cauce Editorial.

Croucher, S. (2004). Globalization and belonging: The politics of identity in a changing world. Lanham: Rowman \& Littlefield Publishers.

Cummins, J. (1986). Empowering minority students: A framework for intervention. Harvard Educational Review, 56, 13-86

De La Torre, I. (2003). Los fundamentos sociales del Tercer Sector. Revista Internacional de Sociología, 35, 45-65.

Desimore, L. (1999). Linking parent involvement with student achievement: do race and income matter? Journal of Educational Research, 93 (1), 11-30.

Fernández Prados, J.S; Navas, M.S.; García, M.C; Sánchez, J.; Pumares, P. y Rojas, A.J. (2002). El asociacionismo inmigrante en Andalucía: diferentes perspectivas. En L. Serra (Coord.) II Seminario de Investigación de la Inmigración Extranjera en Andalucía. 
Sevilla: Dirección General de Coordinación de Políticas Migratorias. Consejería de Gobernación de la Junta de Andalucía.

Flecha, R. y Puigvert, L. (2002). Las comunidades de aprendizaje: Una apuesta por la igualdad educativa. REXE - Estudios y EXperiencias en Educación, vol. 1, n. 1., 11-20.

García Llamas, J.L (2005). Educación Intercultural. Análisis y propuestas. Revista de Educación, 336, 89-109.

González Jiménez, A (2005). El desarrollo de la ciudadanía activa, crítica e intercultural mediante la participación de alumnado en las asociaciones, ONG'S y fundaciones. En E. Soriano (Coord.), La interculturalidad como factor de calidad educativa. Madrid: La Muralla.

Greene, M. (1986). Reflection and passion in teaching. Journal of Curriculum and Supervision, 2, 68-71.

Habermas, J. (1999). La inclusión del otro. Estudios de teoría política. Barcelona: Paidos

Hegedus, Z. (2000). Il presente e'lavvenire: nuove pratiche e nuove rappresentaziones sociale. Milan: F. Angeli. Cop.

Henderson, A.T y Mapp, K. L. (2002). A new wave of evidence: the impact of school, family, and community connections of student achievement. Austin, TX: Soothwest Educational Development Laboratory.

Jordán, J.A (2003). Educar para la convivencia intercultural en sociedades multiculturales. Revista de Educación, Número Extraordinario, 213-239.

Ley Orgánica 1/2002, de 2 de Marzo, reguladora del Derecho a la Asociación, (BOE del 28 de marzo de 2002).

Marban, V. (2003). Sociedad Civil, Tercer Sector y Entidades de acción social en España. Revista Internacional de Sociología, 30, 35-48.

Mezzana, M. (2003). Inmigración y multiculturalidad. Una reflexión andinoamericana. En M. García, M. Martínez y F. Santoloya (Coords). Integración social y empleo: El programa de Integra Diversidad. Sevilla: Junta de Andalucía.

Ministerio de Trabajo y Asuntos Sociales (2008). Extranjeros con certificado de registro o tarjeta de residencia en vigor a 31 de diciembre de 2007. Madrid: Secretaría de Estado de Inmigración y Emigración.

Ministerio de Trabajo e Inmigración (2009). Extranjeros con certificado de registro o tarjeta de residencia en vigor a 31 de diciembre de 2008. Madrid: Secretaria de Estado de Inmigración y Emigración.

Perez, L y López, J (2003). El Tercer Sector en España. Madrid: Ministerio de Trabajo y Asuntos Sociales.

Portes, P. (1999). Social and psychological factors in the academic achievement of children of immigrants: A cultural history puzzle. American Educational Research Journal, 36 (3), 489-507.

Sánchez, R. (2003). La situación del Tercer Sector en España. En J. Piñar y R. Sánchez. (Coord.). El Tercer Sector Iberamericano: Fundaciones, asociaciones y ONG'S. Valencia: Temas de Fundaciones.

Sipi, R. (2000). Las asociaciones de mujeres, ¿agentes de integración social?. Papers, 60, 355-364.

Sleeter, Ch. (1991). Empowerment through Multicultural Education. NY: State University of New York Press.

Shor, I y Freire, P (1987). What is the "dialogical method" of teaching?. Journal of Education, 169, 11-31.

Soriano, E. (Coord.) (2004). La práctica educativa intercultural. Madrid: La Muralla

Soriano, E. (Coord.) (2006). La mujer en la perspectiva intercultural. Madrid: La Muralla

Soriano, E. (Coord.) (2005). Calidad educativa en la escuela intercultural. Almería: Servicio de Publicaciones de la Universidad de Almería.

Soriano, E. (2007). Convivir entre culturas. Un compromiso educativo. En E. Soriano 
(Coord)., Educación para la convivencia intercultural. Madrid: La Muralla.

Soriano, E. (2008). Formando ciudadanos para ejercer la democracia. En E. Soriano (Coord.), Educar para la ciudadanía intercultural y democrática. Madrid: La Muralla.

Vila, I. (2004). La dimensión social y comunitaria de la educación. Trabajadores/as de la Enseñanza. 249, 34-35. www.fe.ccoo.es/publicaciones/TE/249/249.p df . Consultado el 5/4/2008.

\section{Notas}

[1] Proyecto de investigación "Invernaderos e inmigrantes. El problema de adaptación de un colectivo marginal”. Dirección General de Migraciones. Ministerio de Asuntos Sociales.

[2] Proyecto: Etablissement et Evaluation de Programes d`Educación Multiculturelle. Commission des Communautes Europeennes. Direction Genera- les XXII- Education, Formation et Jeunesse. SOCRATES.

[3] Proyecto: La situación de la inmigración en Andalucía. Consejo Económico y Social de Andalucía.

[4] Proyecto en red "Expectativas, realidad social e integración del colectivo inmigrante senegalés en Andaucía. Consejería de Gobernación. Junta de Andalucía.

[5] AIS2, segundo inmigrante entrevistado de la Asociación de Senegal.

[6] AIP1, primer inmigrante entrevistado de la Asociación de Pakistán.

[7] AIR3, tercer entrevistado perteneciente a una asociación rusa.

[8] AIM2, segunda entrevistada de una asociación de inmigrantes marroquíes

[9] P representa al profesor y el número se lo asignamos al realizarle la entrevista. En este caso P5, es el quinto profesor entrevistado.

[10] D hace referencia a un director entrevistado, el número es el que se le asigna al realizarle la entrevista

\section{ABOUT THE AUTHORS / SOBRE LOS AUTORES}

Soriano-Ayala, Encarnación (esoriano@ual.es). Doctora en Pedagogía y profesora de la Universidad de Almería (España). Especializada en educación intercultural, tema sobre el que ha publicado la mayoría de sus trabajos. Es la autora de contacto para este artículo. Su dirección postal es: Área de Métodos de Investigación y Diagnóstico en Educación. Departamento de Ciencias Humanas y Sociales. La Cañada de San Urbano-04120. Almería (España). Buscar otros artículos de esta autora en Google Académico /Find other articles by this author in Scholar Google

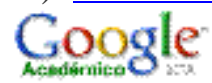

González-Jiménez, Antonio J. (ajgonzal@ual.es). Profesor ayudante en la Universidad de Almería (España). Su dirección postal es: Área de Métodos de Investigación y Diagnóstico en Educación. Departamento de Ciencias Humanas y Sociales. La Cañada de San Urbano-04120. Almería (España). 
Soriano-Ayala, Encarnación \& González-Jiménez, Antonio J. (2010). El poder educativo de las asociaciones de inmigrantes en las escuelas multiculturales. RELIEVE, v. 16, n. 1, p. 1-20. http://www.uv.es/RELIEVE/v16n1/RELIEVEv16n1_3.htm

\title{
ARTICLE RECORD / FICHA DEL ARTÍCULO
}

\begin{tabular}{|c|c|}
\hline $\begin{array}{l}\text { Reference / } \\
\text { Referencia }\end{array}$ & $\begin{array}{l}\text { Soriano-Ayala, Encarnación \& González-Jiménez, Antonio J. (2010). El poder educativo de las asociaciones de } \\
\text { inmigrantes en las escuelas multiculturales. RELIEVE, v. 16, n. 1. } \\
\text { http://www.uv.es/RELIEVE/v16n1/RELIEVEv16n1_3.htm. }\end{array}$ \\
\hline Title / Título & $\begin{array}{l}\text { El poder educativo de las asociaciones de inmigrantes en las escuelas multiculturales. [The education power of } \\
\text { immigrant associations in multicultural schools]. }\end{array}$ \\
\hline \begin{tabular}{|l|} 
Authors / \\
Autores
\end{tabular} & Soriano-Ayala, Encarnación \& González-Jiménez, Antonio J. \\
\hline $\begin{array}{l}\text { Review / } \\
\text { Revista }\end{array}$ & $\begin{array}{l}\text { RELIEVE (Revista ELectrónica de Investigación y EValuación Educativa / E-Journal of Educational Research, } \\
\text { Assessment and Evaluation), v. 16, n. } 1 .\end{array}$ \\
\hline ISSN & $1134-4032$ \\
\hline $\begin{array}{l}\text { Publication } \\
\text { date / } \\
\text { Fecha de } \\
\text { publicación }\end{array}$ & 2009 (Reception Date: 2009 October 08; Approval Date: 2010 April 08; Publication Date: 2010 April 08). \\
\hline $\begin{array}{l}\text { Abstract / } \\
\text { Resumen }\end{array}$ & $\begin{array}{l}\text { With this qualitative research we are trying to know the collaborative capacity and contributions that Immigrant } \\
\text { Associations could do to the schools that form part of a social context characterised by the recent and massive } \\
\text { arrival of immigrants of Maghrebian, Sub-Sahara, South America, European Union, and East Europe origin. The } \\
\text { sample constituted by } 55 \text { immigrants that are members of Immigrant Associations, } 16 \text { teachers and } 16 \text { directors } \\
\text { of schools, makes us to think about the role the Immigrant Associations could play in the education centres. The } \\
\text { information coming out from immigrants and teachers shows up that the participation of associations, besides to } \\
\text { do a good intercultural work and favour the identity signs of new students, empower the school influence of im- } \\
\text { migrant children. In addition to, this participation would support the continuity between the school and the stu- } \\
\text { dent family; it also constitutes a way of working with children and young people in communities of learning. } \\
\text { Con esta investigación cualitativa pretendemos dar a conocer la capacidad colaborativa y las aportaciones que } \\
\text { pueden realizar las asociaciones de inmigrantes a las escuelas que forman parte de un contexto social caracteriza- } \\
\text { do por la llegada reciente y masiva de inmigrantes de orígenes Magrebí, África Subsahariana, Sudamérica, Unión } \\
\text { Europea y Europa del Este. La muestra constituida por } 55 \text { inmigrantes miembros de asociaciones de inmigrantes } \\
\text { y } 16 \text { profesores y } 16 \text { directores de centros educativos, nos hace reflexionar sobre el papel que pueden jugar las } \\
\text { asociaciones de inmigrantes en los centros educativos. Los datos obtenidos a partir de los propios inmigrantes y } \\
\text { profesores, ponen de manifiesto que la participación de las asociaciones, además de realizar una buena labor de } \\
\text { mediación intercultural y favorecer las señas identitarias de los nuevos escolares, potencia el “poder” escolar y } \\
\text { social de niños y jóvenes inmigrantes. Además, esta participación apoyaría la continuidad entre centro educativo } \\
\text { y familias, y constituye una forma de poder trabajar con los niños y jóvenes en comunidades de aprendizaje. }\end{array}$ \\
\hline $\begin{array}{l}\text { Keywords / } \\
\text { Descriptores }\end{array}$ & $\begin{array}{l}\text { Immigrant Associations, Intercultural Mediation, Empowerment, communities of learning. } \\
\text { Asociaciones de inmigrantes, mediación intercultural, empoderamiento, comunidades de aprendizaje }\end{array}$ \\
\hline $\begin{array}{l}\text { Institution / } \\
\text { Institución }\end{array}$ & Universidad de Almería (Spain). \\
\hline $\begin{array}{l}\text { Publication site } \\
\text { / Dirección }\end{array}$ & http://www.uv.es/RELIEVE \\
\hline $\begin{array}{l}\text { Language / } \\
\text { Idioma }\end{array}$ & Español (Title, abstract and keywords in English) \\
\hline
\end{tabular}

\section{RELIEVE}

\section{Revista ELectrónica de Investigación y EValuación Educativa E-Journal of Educational Research, Assessment and Evaluation}

[ISSN: 1134-4032]

\begin{abstract}
(C) Copyright, RELIEVE. Reproduction and distribution of this articles it is authorized if the content is no modified and their origin is indicated (RELIEVE Journal, volume, number and electronic address of the document).

(C) Copyright, RELIEVE. Se autoriza la reproducción y distribución de este artículo siempre que no se modifique el contenido y se indique su origen (RELIEVE, volumen, número y dirección electrónica del documento).
\end{abstract}

